\title{
DISTRIBUCIÓN ESPACIAL DE LOS ACCIDENTES Y ENFERMEDADES RELACIONADOS CON EL TRABAJO EN EL PERÚ, 2012-2014
}

\author{
Akram Hernández-Vásquez ${ }^{1, a}$, Deysi Díaz-Seijas ${ }^{2, b}$, Stalin Vilcarromero ${ }^{3, c}$, Marilina Santero ${ }^{1, d}$
}

\begin{abstract}
RESUMEN
Se analizaron geoespacialmente los accidentes, incidentes peligrosos y enfermedades ocupacionales que se reportaron a nivel regional en el Perú (2012-2014). 52887 eventos se notificaron entre accidentes de trabajo (93\%), incidentes peligrosos $(5,1 \%)$, enfermedades ocupacionales $(1 \%)$ y accidentes mortales $(0,9 \%)$. Se evidenciaron altas tasas de accidentes mortales en Pasco, Callao, Lima, Moquegua y Arequipa. Callao y Lima son las regiones con tasas más altas de accidentes de trabajo. Las mayores tasas de incidentes peligrosos se reportaron en Arequipa, Callao, Lima, Ica y Piura. Las enfermedades ocupacionales se distribuyeron con altas tasas en Huancavelica, Ancash, Pasco, Callao y Cusco. La explotación de minas y canteras $(49,2 \%)$; seguida por la industria manufacturera $(23,4 \%)$; y, la construcción $(8 \%)$ son las actividades económicas que concentraron elevadas tasas de enfermedades ocupacionales. Se concluye que existen altas tasas y patrones espaciales comunes de accidentabilidad en el Perú que pueden servir para enfocar intervenciones.
\end{abstract}

Palabras clave: Sistemas de información geográfica; Salud laboral; Accidentes de trabajo; Enfermedades profesionales (fuente: DeCS BIREME).

\section{SPATIAL DISTRIBUTION OF ACCIDENTS, INCIDENTS AND DISEASES RELATED TO WORK IN PERU, 2012-2014}

\begin{abstract}
We analyzed geospatially accidents, incidents and diseases related to work of regional reports in Peru (2012-2014). The 52887 events were classified as work related accidents (93\%), dangerous incidents $(5.1 \%)$, occupational diseases $(1 \%)$ and fatal accidents $(0.9 \%)$. The highest rates of fatal accidents were reported in Pasco, Callao, Lima, Moquegua and Arequipa. Callao and Lima are the regions with the highest rates of occupational accidents. The highest rates of dangerous incidents were reported in Arequipa, Callao, Lima, Ica and Piura. Occupational diseases are distributed with high rates in Huancavelica, Ancash, Pasco, Callao and Cusco. The economic activities that reported most of the occupational diseases were mining and quarrying (49.2\%); followed by manufacturing industry (23.4\%); and construction $(8 \%)$. It is concluded that there are high rates and common spatial patterns of laboral accidents in Peru that could be used by decision makers to focus interventions.
\end{abstract}

Key words: Geographic information systems; Occupational health; Accidents, occupational; Occupational diseases (source: MeSH NLM).

\section{INTRODUCCIÓN}

La fuerza laboral de un país es el pilar para el desarrollo económico, es por ello que diversas instituciones y organismos internacionales han realizado un llamado para mejorar el estado de salud de los trabajadores (1). Sin embargo, las condiciones laborales de algunos países en desarrollo crean una serie de riesgos para los trabajadores y generan una carga de enfermedad que puede convertirse en un importante problema de salud pública ${ }^{(2)}$. Es así que la ocurrencia de accidentes y enfermedades del trabajo genera daños físicos, psicológicos y sociales a las víctimas llegando, incluso, a ser letales. Por lo tanto, prevenir o reducir su ocurrencia

\footnotetext{
Universidad de Buenos Aires. Buenos Aires, Argentina.

Instituto Nacional Cardiovascular, EsSalud. Lima, Perú.

Sociedad Científica de Estudiantes de Medicina de la Amazonía Peruana. Facultad de Medicina, Universidad Nacional de la Amazonía Peruana. Iquitos, Perú.

Médico cirujano, magíster en Gestión y Políticas Públicas; ${ }^{\mathrm{b}}$ enfermera, especialista en Cuidados Intensivos; ${ }^{\mathrm{c}}$ médico cirujano, magíster en Salud Pública; ${ }^{\mathrm{d}}$ médica

El presente estudio ha sido presentado al XIV Congreso Latinoamericano de Salud Ocupacional.

Recibido: 20-10-15 Aprobado: 09-03-16
}

Citar como: Hernández-Vásquez A, Díaz-Seijas D, Vilcarromero S, Santero M. Distribución espacial de los accidentes y enfermedades relacionados con el trabajo en el Perú, 2012-2014. Rev Peru Med Exp Salud Publica. 2016;33(1):106-12. doi: 10.17843/rpmesp.2016.331.2013 
y mitigar su impacto es uno de los objetivos de la salud y seguridad en el trabajo.

En el Perú, con la aprobación de la Ley 29783 en el año 2011, se establece la notificación obligatoria de los accidentes de trabajo y enfermedades ocupacionales al Ministerio de Trabajo y Promoción del Empleo (MTPE), lo que representa un punto de partida y oportunidad para la recopilación de datos y análisis epidemiológicos que orienten la implementación de programas y políticas laborales ${ }^{(3)}$.

Por otro lado, la diversidad geográfica y socioeconómica, imprime de manera significativa grandes variaciones y desigualdades territoriales a la salud poblacional. Es por ello, que en los últimos años existe una tendencia a desarrollar investigaciones con una perspectiva espacial que incorporen la variabilidad territorial al estudio de los problemas de salud mediante los Sistemas de Información Geográfica (SIG) ${ }^{(4)}$. Los SIG son producto de las ciencias informáticas y geográficas que tienen muchos componentes, pero cuyo propósito es visualizar el problema de salud y otros determinantes según su dimensión espacial, de modo que permita determinar la situación actual y oriente la toma de decisiones ${ }^{(5)}$. No obstante, en el Perú aún no se han publicado investigaciones en temas de salud ocupacional (SO) que incorporen estas herramientas.

Por este motivo, el presente estudio tuvo como objetivo describir y proporcionar una representación geoespacial de las tasas de accidentes mortales, accidentes de trabajo, incidentes peligrosos y enfermedades ocupacionales en el Perú haciendo énfasis en las actividades económicas que concentran el mayor número enfermedades ocupacionales.

\section{EL ESTUDIO}

Se realizó un estudio exploratorio ecológico de distribución espacial de accidentes mortales, accidentes de trabajo, incidentes peligrosos y enfermedades ocupacionales tomando como unidad de análisis las 25 regiones del Perú durante el periodo 2012-2014. Las variables en estudio han sido definidas por el MTPE como: a). Accidente mortal.- lesión que genera la muerte del trabajador, sin tomar en cuenta el tiempo transcurrido entre la fecha del accidente y el deceso; b). Accidente de trabajo.- suceso repentino que sobreviene por causa o con ocasión del trabajo y que le puede ocasionar al trabajador una lesión orgánica, una perturbación funcional, una invalidez o la muerte; c). Incidentes peligrosos.- todo suceso potencialmente riesgoso que pudiera causar lesiones o enfermedades a las personas en su trabajo o a la población; y d). Enfermedades ocupacionales.- enfermedad contraída como resultado de la exposición a factores de riesgo como agentes físicos, químicos, biológicos y ergonómicos, inherentes a la actividad laboral (6).

En una primera fase se obtuvieron los reportes regionales, de los anuarios estadísticos del 2012 al 2014, de accidentes mortales, accidentes de trabajo, incidentes peligrosos y enfermedades ocupacionales publicados en el portal web del MTPE (http://www. mintra.gob. pe/mostrarContenido. php?id=86\&tip=87). Seguidamente, se obtuvieron del sitio web del Instituto Nacional de Estadística e Informática (INEI) los reportes de la población económicamente activa ocupada (PEAO) según ámbito geográfico para los años de estudio (http://www.inei.gob.pe/estadisticas/ indice-tematico/ocupacion-y-vivienda/). Se estimaron tasas específicas entre el número anual de accidentes mortales, accidentes de trabajo, incidentes peligrosos $y$ enfermedades ocupacionales, y el total de la PEAO por cada 100000 personas económicamente activas ocupadas en cada región. Finalmente, las enfermedades ocupacionales reportadas fueron categorizadas según actividad económica mediante tasas regionales de ocurrencia. Las tasas obtenidas se incluyeron en una hoja de cálculo de Microsoft Excel ${ }^{\circledR} 2013$ y dos investigadores realizaron el control de calidad final.

Una segunda fase consistió en el análisis exploratorio de datos espaciales a partir de la integración de las tasas registradas en la hoja de cálculo a la cartografía obtenida del portal web del Ministerio del Ambiente. Como último paso, se usó el software QGIS v2.10.1 (OSGeo, Beaverton, OR, USA) para procesar, analizar y elaborar los mapas temáticos finales agrupando las regiones en quintiles.

El presente estudio no requirió la aprobación de un comité de ética por tratarse de un análisis de datos secundarios obtenidos de un dominio público y de libre acceso que no permite identificar a los sujetos cuyos casos fueron reportados.

\section{HALLAZGOS}

Un total de 52887 eventos fueron reportados al MTPE desde enero 2012 a diciembre 2014, correspondiendo el $93 \%$ a accidentes de trabajo seguido por incidentes peligrosos $(5,1 \%)$, enfermedades ocupacionales $(1 \%)$ y accidentes mortales $(0,9 \%)$. La Tabla 1 resume el número de eventos reportados y las tasas regionales calculadas por 100000 personas de la PEAO. 
Tabla 1. Número de eventos reportados por regiones y tasas durante el periodo 2012-2014, Perú

\begin{tabular}{|c|c|c|c|c|c|c|c|c|}
\hline \multirow[b]{2}{*}{ Regiones } & \multirow{2}{*}{$\begin{array}{c}\text { Accidentes } \\
\text { mortales }\end{array}$} & \multirow{2}{*}{$\begin{array}{c}\text { Accidentes } \\
\text { de trabajo }\end{array}$} & \multirow{2}{*}{$\begin{array}{l}\text { Incidentes } \\
\text { peligrosos }\end{array}$} & \multirow{2}{*}{$\begin{array}{l}\text { Enfermedades } \\
\text { ocupacionales }\end{array}$} & \multicolumn{4}{|c|}{ Tasa por 100000 personas ocupadas } \\
\hline & & & & & $\begin{array}{l}\text { Accidentes } \\
\text { mortales }\end{array}$ & $\begin{array}{c}\text { Accidentes } \\
\text { de trabajo }\end{array}$ & $\begin{array}{l}\text { Incidentes } \\
\text { peligrosos }\end{array}$ & $\begin{array}{l}\text { Enfermedades } \\
\text { ocupacionales }\end{array}$ \\
\hline Amazonas & 0 & 3 & 0 & 0 & 0,00 & 0,45 & 0,00 & 0,00 \\
\hline Ancash & 17 & 186 & 38 & 63 & 0,94 & 10,32 & 2,11 & 3,50 \\
\hline Apurímac & 1 & 3 & 1 & 0 & 0,13 & 0,40 & 0,13 & 0,00 \\
\hline Arequipa & 31 & 1746 & 365 & 44 & 1,58 & 88,74 & 18,55 & 2,24 \\
\hline Ayacucho & 8 & 20 & 10 & 2 & 0,78 & 1,94 & 0,97 & 0,19 \\
\hline Cajamarca & 12 & 46 & 12 & 0 & 0,51 & 1,95 & 0,51 & 0,00 \\
\hline Callao & 29 & 7715 & 189 & 40 & 1,94 & 515,75 & 12,63 & 2,67 \\
\hline Cusco & 3 & 372 & 8 & 51 & 0,14 & 16,91 & 0,36 & 2,32 \\
\hline Huancavelica & 3 & 59 & 0 & 59 & 0,39 & 7,68 & 0,00 & 7,68 \\
\hline Huánuco & 4 & 7 & 11 & 0 & 0,30 & 0,53 & 0,83 & 0,00 \\
\hline Ica & 8 & 90 & 96 & 4 & 0,66 & 7,48 & 7,98 & 0,33 \\
\hline Junín & 9 & 97 & 30 & 0 & 0,44 & 4,73 & 1,46 & 0,00 \\
\hline La Libertad & 20 & 176 & 92 & 0 & 0,73 & 6,44 & 3,36 & 0,00 \\
\hline Lambayeque & 13 & 29 & 38 & 0 & 0,71 & 1,59 & 2,09 & 0,00 \\
\hline Lima & 258 & 36454 & 1590 & 242 & 1,78 & 251,02 & 10,95 & 1,67 \\
\hline Loreto & 10 & 566 & 9 & 4 & 0,68 & 38,44 & 0,61 & 0,27 \\
\hline Madre de Dios & 0 & 1 & 2 & 0 & 0,00 & 0,45 & 0,89 & 0,00 \\
\hline Moquegua & 5 & 221 & 9 & 0 & 1,67 & 73,98 & 3,01 & 0,00 \\
\hline Pasco & 9 & 11 & 10 & 13 & 1,98 & 2,42 & 2,20 & 2,86 \\
\hline Piura & 34 & 1231 & 133 & 0 & 1,28 & 46,49 & 5,02 & 0,00 \\
\hline Puno & 5 & 18 & 11 & 0 & 0,21 & 0,76 & 0,47 & 0,00 \\
\hline San Martín & 3 & 5 & 4 & 0 & 0,23 & 0,39 & 0,31 & 0,00 \\
\hline Tacna & 6 & 82 & 5 & 0 & 1,14 & 15,60 & 0,95 & 0,00 \\
\hline Tumbes & 2 & 25 & 14 & 0 & 0,54 & 6,70 & 3,75 & 0,00 \\
\hline Ucayali & 5 & 18 & 12 & 0 & 0,62 & 2,25 & 1,50 & 0,00 \\
\hline
\end{tabular}

Durante el periodo 2012-2014 se reportaron 495 muertes como consecuencia de accidentes de trabajo. En la Figura $1 \mathrm{~A}$ se puede observar en escala de colores las tasas de accidentes mortales para cada región. Los valores más altos corresponden a región Pasco, Callao, Lima, Moquegua y Arequipa. Amazonas y Madre de Dios no reportaron ningún accidente mortal en ese periodo.

Respecto a los accidentes de trabajo, existe una gran variabilidad en las tasas regionales. La distribución espacial de estos eventos permite apreciar en la Figura 1B que Callao y Lima son las regiones con las tasas más elevadas mientras que la más baja corresponde a San Martín seguida de Apurímac, Madre de Dios, Amazonas y Huánuco.

Los incidentes peligrosos reportados fueron 2689 distribuyéndose las mayores tasas en regiones de la costa peruana (Arequipa, Callao, Lima, Ica y Piura) tal como lo muestra la Figura 2A. Las menores tasas reportadas corresponden a Amazonas, Huancavelica, Apurímac, San Martín y Cusco.

En cuanto a las enfermedades ocupacionales, se notificaron 522 entre todas las regiones. La mayor intensidad del color en la Figura 2B muestra que Huancavelica, Ancash, Pasco, Callao y Cusco son las regiones con las más altas tasas. No reportaron enfermedades ocupacionales en quince regiones durante los años de estudio.

A manera general, la distribución espacial de las tasas de eventos reportados identifica un agrupamiento de regiones con altas tasas representadas por Lima, Callao, Arequipa, Piura y Pasco. A nivel de la región natural de la sierra, Pasco tiene un mayor reporte de accidentes mortales y enfermedades ocupacionales. En la región natural de la selva, Loreto presentó las tasas más altas de accidentes mortales, accidentes laborales y enfermedades ocupacionales. 

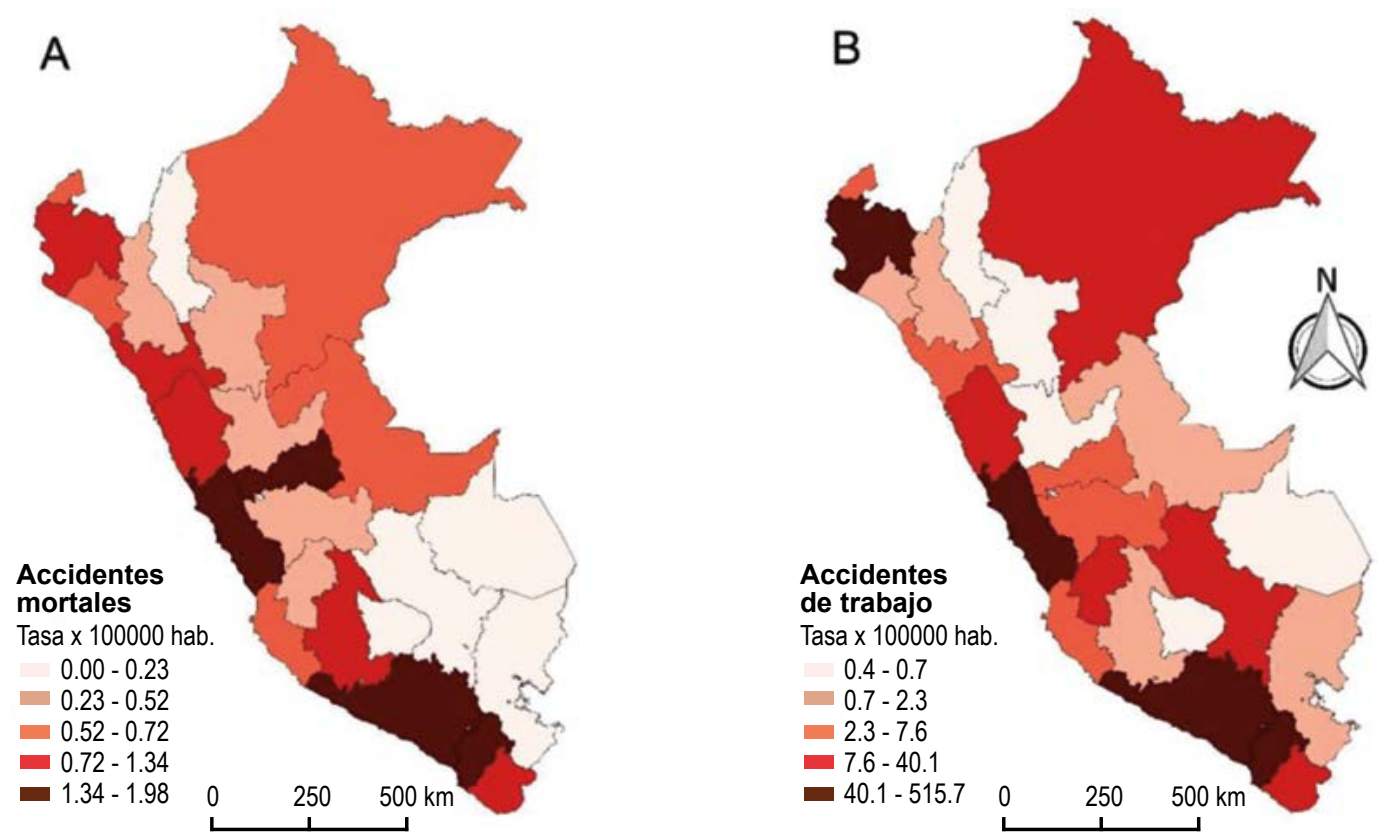

Figura 1. A. Tasa de accidentes mortales durante el periodo 2012-2014, Perú. B. Tasa de accidentes de trabajo durante el periodo 2012-2014, Perú
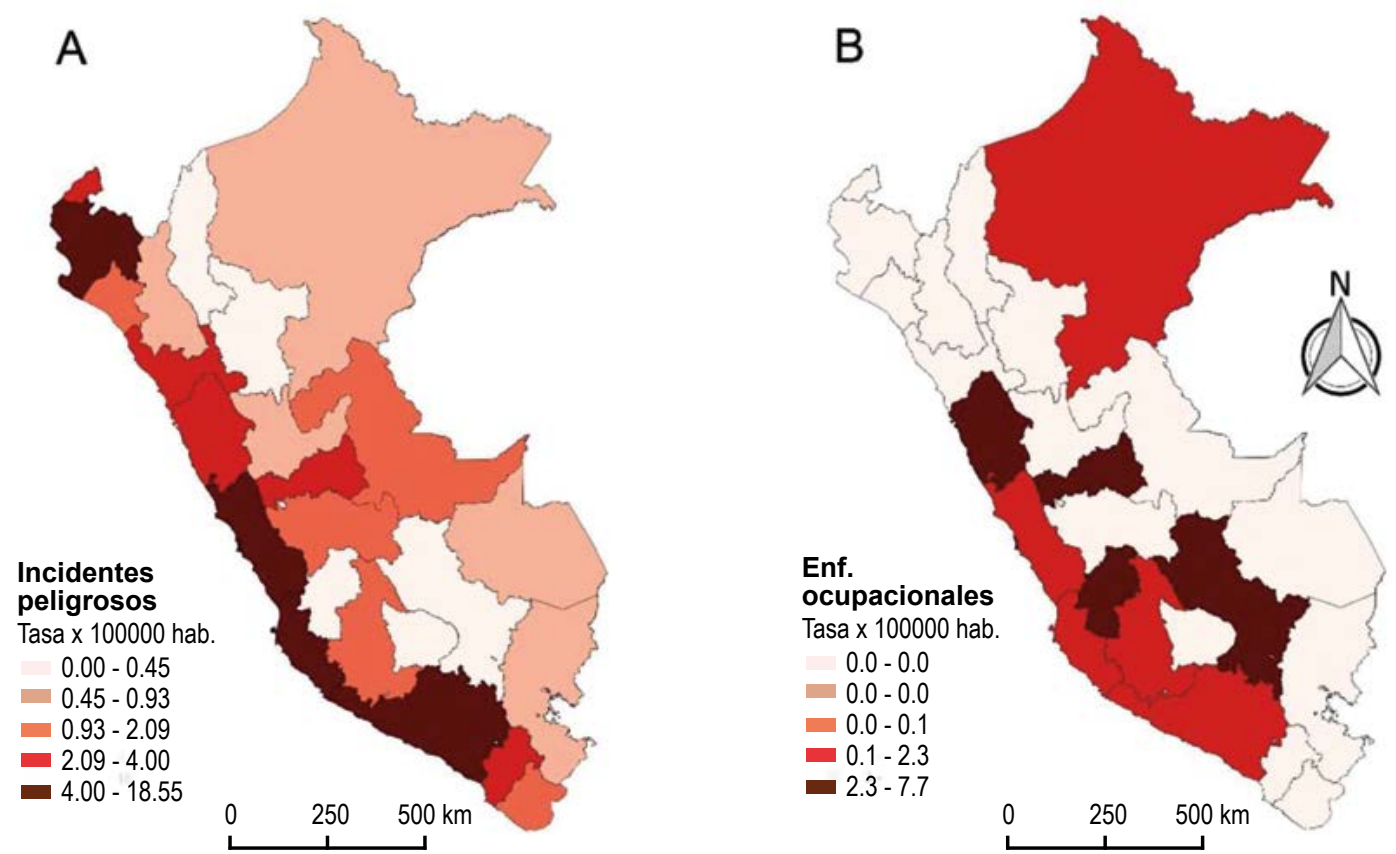

Figura 2. A. Tasa de incidentes peligrosos durante el periodo 2012-2014, Perú. B. Tasa de enfermedades ocupacionales durante el periodo 2012-2014, Perú

Respecto a la concentración de las enfermedades ocupacionales según actividad económica, se obtuvo que para la explotación de minas y canteras se reportaron el mayor número de enfermedades ocupacionales $(49,2 \%)$ con altas tasas en Huancavelica,
Ancash y Pasco; seguida por las actividades de la industria manufacturera $(23,4 \%)$ siendo Callao, Lima e Ica las únicas regiones que reportaron este tipo de eventos; y, las actividades de construcción (8\%) para Lima y Ancash (Tabla 2). 
Tabla 2. Tasas regionales de enfermedades ocupacionales reportadas según actividad económica durante el periodo 2012-2014, Perú

\begin{tabular}{|c|c|c|c|c|c|}
\hline \multirow[b]{2}{*}{ Regiones } & \multicolumn{5}{|c|}{ Tasa por 100000 personas ocupadas } \\
\hline & $\begin{array}{l}\text { Explotación } \\
\text { de minas y } \\
\text { canteras }\end{array}$ & $\begin{array}{c}\text { Industrias } \\
\text { manufactureras }\end{array}$ & Construcción & $\begin{array}{c}\text { Transporte, } \\
\text { almacenamiento y } \\
\text { comunicaciones }\end{array}$ & $\begin{array}{c}\text { Actividades } \\
\text { inmobiliarias, } \\
\text { empresariales y } \\
\text { de alquiler }\end{array}$ \\
\hline Ancash & 2,94 & 0,00 & 0,33 & 0,00 & 0,11 \\
\hline Arequipa & 2,13 & 0,00 & 0,00 & 0,10 & 0,00 \\
\hline Callao & 0,00 & 1,67 & 0,00 & 0,27 & 0,07 \\
\hline Cusco & 1,82 & 0,00 & 0,00 & 0,00 & 0,05 \\
\hline Huancavelica & 7,55 & 0,00 & 0,00 & 0,00 & 0,00 \\
\hline Ica & 0,00 & 0,33 & 0,00 & 0,00 & 0,00 \\
\hline Lima & 0,32 & 0,64 & 0,25 & 0,10 & 0,18 \\
\hline Loreto & 0,27 & 0,00 & 0,00 & 0,00 & 0,00 \\
\hline Pasco & 2,86 & 0,00 & 0,00 & 0,00 & 0,00 \\
\hline
\end{tabular}

\section{DISCUSIÓN}

La vigilancia en SO es crucial para la prevención, reconocimiento y manejo de enfermedades y lesiones en los entornos de trabajo que trae beneficios individuales y colectivos ante la exposición a factores de riesgos ocupacionales ${ }^{(7)}$. Es así que la obligatoriedad para la notificación de los accidentes de trabajo y enfermedades ocupacionales se convierte en una ventana de oportunidad para el desarrollo de estudios que incorporen la territorialidad dentro de las variables de análisis. En general, nuestro estudio encuentra una marcada heterogeneidad interregional a partir de las tasas ajustadas de eventos reportados al MTPE. Asimismo, ejemplifica como los SIG pueden contribuir al estudio de los problemas en SO y que para ello se disponen en la actualidad de software libres como GeoDa, SatScan, gvSIG, TerraView o el QGIS que ha sido usado en este y otros estudios ${ }^{(5)}$.

Está descrito que la competitividad y ubicación geográfica de las actividades económicas cumplen un papel importante en la variabilidad de accidentes y enfermedades ocupacionales desde el punto de vista territorial ${ }^{(8,9)}$. Un estudio basado en datos de la Organización Internacional del Trabajo y del Foro Económico Mundial ha estimado una relación inversa entre competitividad y accidentes ocupacionales, es decir, cuanto menor es el número de accidentes, más alta es la competitividad y la productividad ${ }^{(8)}$. En relación a las actividades económicas regionales, es interesante resaltar que en Lima y Callao predomina la elaboración de productos alimenticios como la actividad manufacturera más importante $(42,9 \%$ y que concentra más del $55 \%$ de las industrias manufactureras ${ }^{(10)}$; mientras que en Arequipa, Pasco y Moquegua predomina la explotación de minas y canteras. Todo lo anterior podría estar relacionado en nuestro estudio por cuanto las regiones con una estructura productiva que genera elevados riesgos como la industria manufacturera, la construcción y la minería presentan elevada accidentabilidad y enfermedades ocupacionales, comparadas con aquellas donde las actividades económicas se orientan más hacia el sector servicios ${ }^{(9)}$, no obstante, la cobertura del Seguro Complementario de Trabajo de Riesgo (SCTR) excluye actividades que, si bien no son consideradas de alto riesgo, presentan elevada accidentabilidad. En consecuencia, se puede esperar que la focalización de actividades preventivo-promocionales junto con procesos de monitoreo y control hacia estas regiones contribuyan a disminuir la carga de enfermedad laboral (11). Además, se debería revaluar la cobertura del SCTR para actividades que no han sido consideradas como de riesgo, de modo que facilite la vigilancia y permita la atención de acuerdo a nuestro contexto ${ }^{(12)}$.

Es importante que el monitoreo de estos eventos se realice en cortos periodos, por los cambios en la fuerza laboral o dinamismo económico territorial que, finalmente, tendrán un impacto en el número de eventos reportados ${ }^{(13)}$. En tal sentido, se deben realizar comparaciones ya sea sobre una base año a año, entre sectores de actividad económica y trabajadores informales con la finalidad de incrementar la exactitud de las estadísticas y disponer de datos fiables. En el presente trabajo se han usado estadísticas oficiales que muchas veces excluyen poblaciones especificas como menores de edad trabajadores o poblaciones subempleadas y que pueden no reflejar la magnitud real 
de las lesiones y enfermedades ocupacionales. A manera de ejemplificar lo anterior, un estudio en Cusco realizado en menores trabajadores mostró una prevalencia de lesiones graves del $3 \%$, las cuales muchas veces ocurrieron bajo condiciones laborales deficientes ${ }^{(14)} \mathrm{y}$, por otro lado, está la alta tasa de trabajo informal que aún fluctúa en más del $50 \%{ }^{(15)}$.

La calidad y grado de las notificaciones sigue siendo un problema en la actualidad. Diversos estudios ponen de manifiesto la falta de información sistematizada, subregistro de eventos y la existencia de múltiples registros que difieren entre sí ${ }^{(16-19)}$. Una reciente publicación utilizó como fuente de datos los registros mensuales del MTPE, a diferencia del presente trabajo que usó los anuarios estadísticos, es decir, existen dos fuentes de información, una desagregada que se actualiza mensualmente y otra consolidada anualmente, en consecuencia, al contrastar los resultados para el periodo de estudio se puede observar diferencias, como en el caso del número de accidentes mortales (505 versus 495 en nuestro estudio) ${ }^{(20)}$.

Este estudio tiene limitaciones, propias de la metodología empleada. Al tratarse de un estudio de registros se pueden tener errores en la codificación de los eventos durante el llenado de los formatos establecidos por el MINTRA para reportar los casos, o es posible que los informantes omitan consignar datos relevantes acerca de las circunstancias que dieron origen a los hechos y su relación de causalidad ${ }^{(12)}$. Desafortunadamente no se tienen reportes distritales o georreferenciados de los eventos para realizar un análisis espacial con una unidad de estudio menor o de asociación espacial, lo que podría enriquecer aun más los resultados y lograr una mayor focalización de los eventos.

En conclusión, este estudio muestra una considerable variabilidad geográfica y patrones espaciales comunes en los indicadores regionales de accidentabilidad $y$ enfermedades ocupacionales que puede servir de guía para el desarrollo de estudios que ayuden a explicar la variabilidad encontrada y así enfocar futuras intervenciones o acciones a nivel regional con el objetivo de proteger la salud y seguridad de los trabajadores en las distintas regiones del Perú.

Contribuciones de autoría: AHV tuvo la idea de investigación. AHV y DDS diseñaron el estudio y recopilaron los datos. AHV realizó el procesamiento espacial de los datos. AHV, DDS, SV y MS analizaron e interpretaron los datos, redactaron el manuscrito y aprobaron la versión final.

Fuentes de financiamiento: autofinanciado.

Conflictos de interés: los autores declaran no tener conflictos de interés.

\section{REFERENCIAS BIBLIOGRÁFICAS}

1. Organización Panamericana de la Salud. Salud de los trabajadores en la región de las Américas. Cuadragésimo Primer Consejo Directivo [Internet]. Ginebra: OPS/OMS; 1999 [Citado el 28 de agosto de 2015]. Disponible en: http://www.who.int/occupational_ health/regions/en/oeh41 consejo. pdf ?ua $=1$.

2. Concha-Barrientos M, Nelson DI, Fingerhut M, Driscoll T, Leigh J. The global burden due to occupational injury. Am J Ind Med. 2005;48(6):47081.

3. Hernandez-Vasquez A, MarcalayaBenites F, Santiani JA, Pretell-Paredes L. [Need for occupational health education in undergraduate medical education]. Rev Peru Med Exp Salud Publica. 2014;31(3):605-6.

4. Robertson H, Nicholas N, Georgiou A, Johnson J, Travaglia J. Globalising health informatics: the role of GIScience. Stud Health Technol Inform. 2014;205:1168-72.
5. Delaunay M, Van der Westhuizen H, Godard V, Agius R, Le Barbier $\mathrm{M}$, Godderis L, et al. Use of GIS in visualization of work-related health problems. Occup Med (Lond). 2015;65(8):682-92. doi: 10.1093/ occmed/kqv152.

6. Ministerio de Trabajo y Promoción del Empleo. Glosario de Accidentes de trabajo [Internet]. Lima, Perú: Ministerio de Trabajo y Promoción del Empleo; 2005 [Citado el 12 de setiembre de 2015]. Disponible en: http://www.mintra.gob. pe/contenidos/archivos/sst/ DS_009_2005_TR_GLOSARIO TRABAJO.pdf.

7. Azaroff LS, Levenstein C, Wegman DH. Occupational injury and illness surveillance: conceptual filters explain underreporting. Am J Public Health. 2002;92(9):1421-9.

8. Takala J, Hamalainen P, Saarela KL, Yun LY, Manickam K, Jin TW, et al. Global estimates of the burden of injury and illness at work in 2012. J Occup
Environ Hyg. 2014;11(5):326-37. doi: 10.1080/15459624.2013.863131.

9. Gonzalez-Delgado M, Gomez-Dantes $\mathrm{H}$, Fernandez-Nino JA, Robles E, Borja VH, Aguilar M. Factors associated with fatal occupational accidents among Mexican workers: a national analysis. PloS One. 2015;10(3):e0121490. doi: 10.1371/ journal.pone.0121490.

10. Cárdenas G. ¿Existe aún la industria manufacturera en el Perú? Pensamiento Crítico. 2014;11:11-32.

11. Levine DI, Toffel MW, Johnson MS. Randomized government safety inspections reduce worker injuries with no detectable job loss. Science. 2012;336(6083):907-11. doi: 10.1126/science.1215191.

12. Cruz I, Huerta-Mercado R. Occupational Safety and Health in Peru. Ann Glob Health. 2015;81(4):568-75. doi: $10.1016 / \mathrm{j}$. aogh.2015.08.027.

13. Mendeloff J, Staetsky L. Occupational fatality risks in the United States 
and the United Kingdom. Am J Ind Med. 2014;57(1):4-14. doi: 10.1002/ ajim.22258.

14. Schlick C, Joachin M, Briceno L, Moraga D, Radon K. Occupational injuries among children and adolescents in Cusco Province: a crosssectional study. BMC Public Health. 2014;14:766. doi: 10.1186/14712458-14-766.

15. International Labour Organization (ILO). Programme for the Promotion of Formalization in Latin America and the Caribbean. Trends in informal employment in Peru: 2004-2012 [Internet]. Switzerland: ILO; 2014 [Citado el 29 de septiembre de 2015]. Disponible en: http://www.ilo.org/ wcmsp5/groups/public/---americas/--ro-lima/documents/publication/ wcms_245891.pdf
16. Stout N, Bell C. Effectiveness of source documents for identifying fatal occupational injuries: a synthesis of studies. Am J Public Health. 1991;81(6):725-8.

17. Centers for Disease Control and Prevention (CDC). Occupational injuries and deaths among younger workers--United States, 1998-2007. MMWR Morb Mortal Wkly Rep. 2010;59(15):449-55.

18. Adanaque-Gomez M, Loro-Gomez A, Purizaca-Rosillo N. [Underreporting of health personnel accidents caused by sharp objects in a hospital of Piura, Peru]. Rev Peru Med Exp Salud Publica. 2014;31(1):169-80.

19. Haro-Garcia L, Aguilar-Madrid G, Juarez-Perez CA, Aguilar-Rodriguez SD, Flores-Carbajal G, Gea-Izquierdo $\mathrm{E}$, et al. [Inequity and health in the workplace: exploring an emergent area]. Rev Peru Med Exp Salud Publica. 2013;30(4):671-5.

20. Mejia CR, Cárdenas MM, GomeroCuadra R. [Notification of accidents and occupational diseases to the Ministry of Labor. Peru 2010-2014]. Rev Peru Med Exp Salud Publica. 2015;32(3):526-31.

Correspondencia: Akram Abdul Hernández Vásquez

Dirección: Calle Gavilán 3856, Ciudad Autónoma de Buenos Aires, Argentina Teléfono: (0054) 91122981572

Correo electrónico: akram.hernandez.v@upch.pe

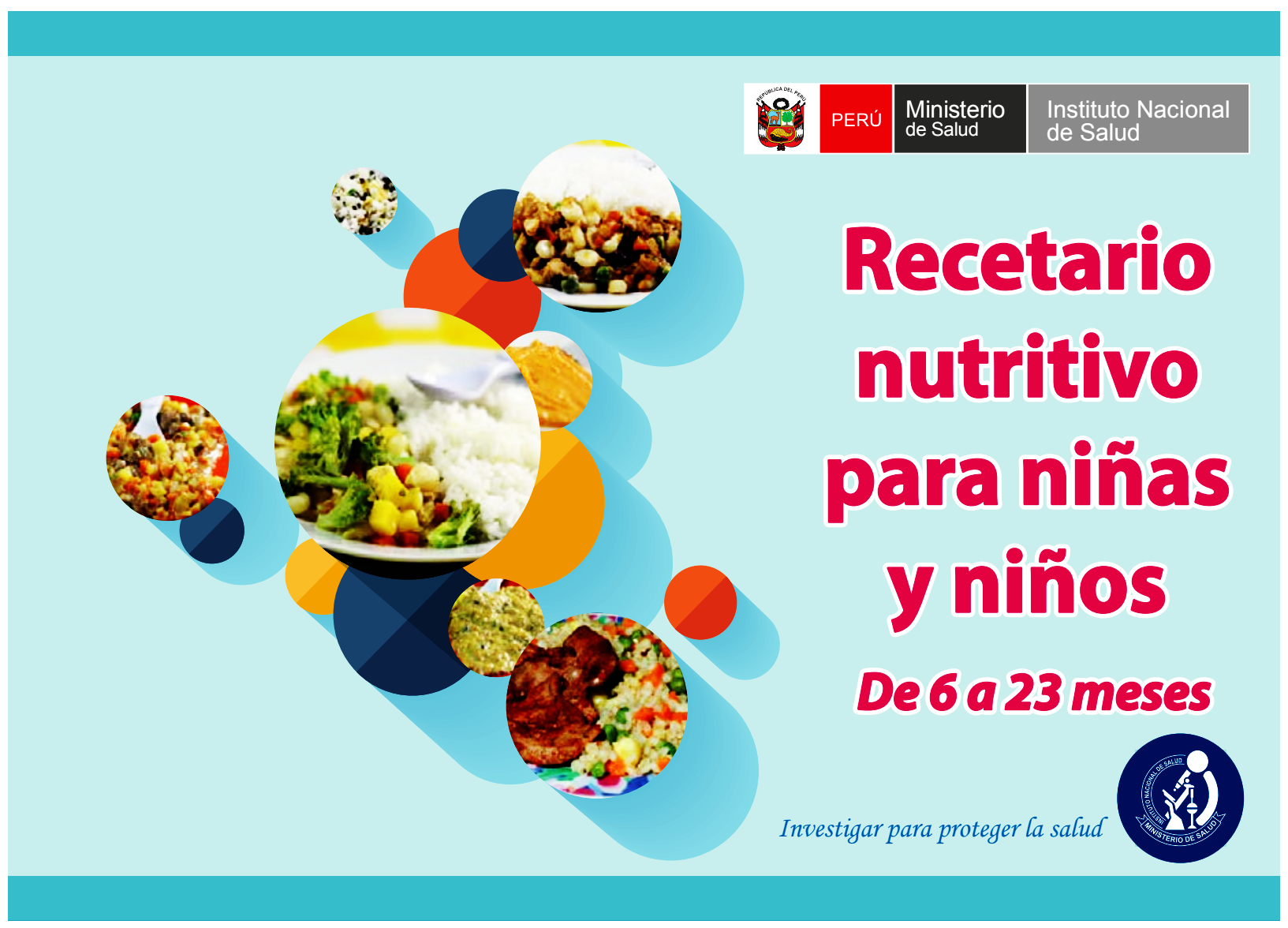

